\title{
Harmful or Helpful? A Systematic Review of How Those Bereaved Through Suicide Experience Research Participation
}

\author{
Karl Andriessen, Karolina Krysinska, Brian Draper, Michael Dudley, and Philip B. Mitchell
}

School of Psychiatry, University of New South Wales, Randwick, NSW, Australia

\begin{abstract}
Background: Many of the bereaved through suicide are interested in participating in postvention studies. However, there is a contradiction between the positive experiences of research participation and concerns raised by ethical boards. Aims: To review studies on the experience of research participation by those bereaved through suicide, including initial contact with the study and its short- and long-term impacts. Method: Systematic searches in Embase, Medline, PsycINFO, as well as Google Scholar identified 12 papers reporting on 11 studies. Results: The majority (73-100\%) of study participants evaluated participation positively, and would recommend it to others (90-100\%), as it was related to altruism, social support, and personal growth. A minority experienced participation as negative (2-10\%) or upsetting (5-22\%) due to feelings of guilt or painful memories. However, having a painful experience does not preclude seeing it as helpful. Limitations: Most studies concerned face-to-face psychological autopsy studies, and only two studies included a control group. Conclusion: Research applying standardized measures may enhance our understanding of the factors germane to (non-)participation and to the likelihood of a positive/negative research experience. Vigilant recruitment and providing optimum care for participants are indicated. Further research may continue to improve participant safety and the research design of suicide bereavement studies.
\end{abstract}

Keywords: bereavement, postvention, research participation, research subjects, suicide

A suicide death can have a devastating and lasting impact on the bereaved family, friends, and others who are affected by the death, to the extent that some may feel that it has changed their lives. Feelings of guilt, abandonment, rejection, and being a part of the "decision" for suicide are frequent among those bereaved through suicide, and some have to cope with consequences related to social functioning (e.g., familial relationships), physical or mental health (e.g., depression, anxiety), or their own suicidal behavior (Grad \& Andriessen, 2016; Pitman, Osborn, King, \& Erlangsen, 2014). There is, however, also evidence of personal growth after a suicide death (Castelli Dransart, 2017; Genest, Moore, \& Nowicke, 2017). Bereaved individuals may learn to cope, make sense, and create new meanings through their grief experiences, a process which may involve individually varying levels of intrapsychic work and social and/or professional support (Neimeyer \& Sands, 2017; Supiano, 2012).

Suicide bereavement support (i.e., postvention), is available in many countries, and has been recognized as an important strategy for suicide prevention (World Health Organization, 2014). There has been significant develop- ment in postvention research over the past decades (Andriessen, Krysinska, \& Grad, 2017; Maple et al., in press), and many of the bereaved are interested in participating in postvention studies (Wilson, 2010). A need to vent and to share the grief experiences, a desire to gain insight and better understanding of the suicide, and willingness to help others may motivate bereaved people to participate in research (Dyregrov, Dieserud, Straiton, et al., 2011). Altruism seems to be a major motivation: The bereaved hope that by taking part in studies they help to prevent suicide and contribute to research progress and better services for the bereaved (Dyregrov, Dieserud, Straiton, et al., 2011). Other benefits of research participation, reported for traumatized populations, include fostering empowerment and self-esteem, strengthening kinship, reducing stigma, normalizing trauma-related reactions, and ensuring safe disclosure of trauma-related information (Newman \& Kaloupek, 2004).

However, there appears to be a contradiction between the accumulating evidence regarding the positive experience of participation in postvention studies (Dyregrov, Dieserud, Hjelmeland, et al., 2011; Henry \& Greenfield, 
2009; Litman, Curphey, Shneidman, Farberow, \& Tabachnick, 1963) and the apparent lack of short-term and longterm negative effects (Hawton, Houston, Malmbergand, \& Simkin, 2003; Wong et al., 2010), on the one hand, and ethical concerns regarding potential harm to research participants (Feigelman, Jordan, McIntosh, \& Feigelman, 2012; Moore, Maple, Mitchell, \& Cerel, 2013; Omerov, Steineck, Dyregrov, Runeson, \& Nyberg, 2014), on the other hand. On the basis of an international survey of researchers, Moore et al. (2013) reported that ethical boards (institutional review boards, ethics committees) are concerned that talking about the grief experience may traumatize and even increase the suicide risk of people bereaved by suicide; moreover, there is lack of certainty that research participants who are negatively impacted will receive sufficient support. Other concerns have been raised in regard to how the suicide-bereaved participants are recruited, how confidentiality and data security can be ensured, and whether there is a risk of coercion related to self-identification as a suicide survivor (Moore et al., 2013).

Similar concerns regarding participant safety and the potential negative impact of research participation have been voiced in suicidology research in general (Dazzi, Gribble, Wessely, \& Fear, 2014; DeCou \& Schumann, 2017; Hom, Podlogar, Stanley, \& Joiner Jr., 2016; Lakeman \& Fitzgerald, 2009a, 2009b), trauma and violence research (McClinton Appollis, Lund, de Vries, \& Mathews, 2015; Newman \& Kaloupek, 2004; Rosenbaum \& Langhinrichsen-Rohling, 2006), bereavement studies (Buckle, Dwyer, \& Jackson, 2010; Cook \& Bosley, 1995; Hynson, Aroni, Bauld, \& Sawyer, 2006; Scarth \& Schafer, 2016), palliative care research (Gysels, Evans, \& Higginson, 2012), and psychiatric research (Jorm, Kelly, \& Morgan, 2007). For instance, Newman and Kaloupek (2004) identified worsening of participant's condition, evoking painful memories and strong emotional distress (e.g., shame, anger and fear), fostering self-destructive behavior, and social risks, such as breach of privacy, risk of social rejection, and social stigma, as major risks of participation in trauma-focused research. These concerns reflect the core values guiding ethics committees internationally (World Medical Association, 2013). For instance, the Australian National Statement on Ethical Conduct in Human Research 2007 (Commonwealth of Australia, 2015) states:

The values of respect, research merit and integrity, justice, and beneficence have become prominent in the ethics of human research in the past six decades, and they provide a substantial and flexible framework for principles to guide the design, review and conduct of such research. (p. 9)

According to the principle of beneficence, "research is ethically acceptable only when its potential benefits justify any risks involved in the research" (Commonwealth of
Australia, 2015, p. 14). Hence, researchers must assess the potential risks in terms of harm or discomfort due to research participation, and they must design the research to prevent, minimize, or manage the risks. Research participation is also voluntary and based on information and understanding of the purpose, requirements, and potential risks and benefits of the research and of participation (World Medical Association, 2013).

To the best of our knowledge, no systematic review has examined the experience of research participation by people bereaved through suicide. The current study aims to redress this gap by reviewing published studies on participants' experiences with research participation, including the initial contact with the study, and its short-term and long-term impacts. The findings of this systematic review can inform members of ethical boards and researchers involved in suicide bereavement studies, as well as those bereaved who may wish to participate in such studies.

\section{Method}

The review was conducted following the PRISMA guidelines (http://www.prisma-statement.org/; Moher, Liberati, Tetzlaff, \& Altman, 2009). Searches were conducted in Embase, Medline, and PsycINFO, with an additional search in Google Scholar. Medline was searched with a combination of MeSH and text words: (research subjects OR research subjects [MeSH] OR human experimentation [MeSH] OR (research AND particip*)) AND (bereavement OR bereavement [MeSH] OR grief OR grief [MeSH]) AND (suicide OR suicide [MeSH]). Subject headings and keywords were used in Embase and PsycINFO. The search, limited to publications in English, was undertaken in June 2017, without restriction for date of publication. Two researchers (KA and $\mathrm{KK}$ ) independently assessed titles and abstracts for eligibility. Any disagreement was resolved through discussion. Potentially relevant studies published in peer-reviewed journals were examined against the inclusion/exclusion criteria. Original studies were included if: (a) the study used quantitative, qualitative, or mixed-methods approaches; (b) the sample consisted of people bereaved through suicide; and (c) the study reported data regarding the experience of research participation. Review articles were excluded. The references of retrieved papers were hand-checked to identify additional studies. Figure 1 depicts the search and selection process. 


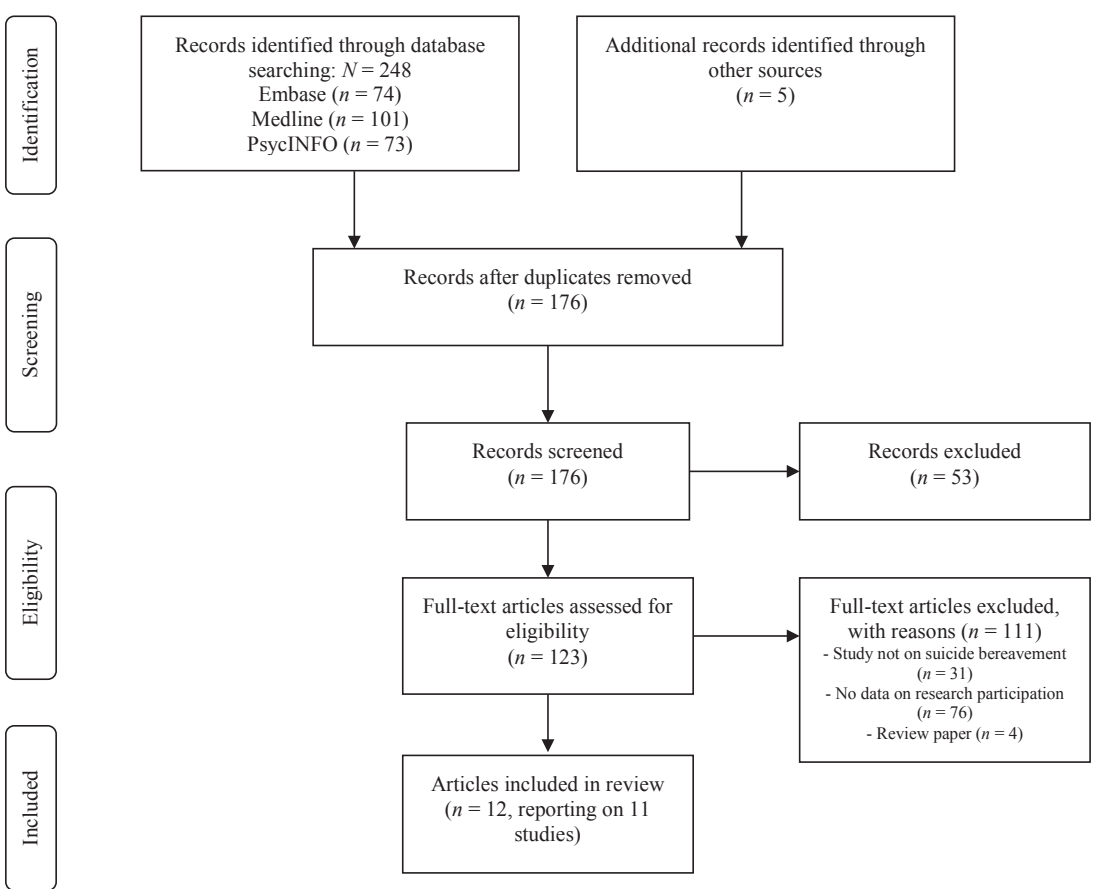

Figure 1. PRISMA flow diagram

\section{Results}

\section{Participants and Research Methodology}

The systematic search identified 12 papers reporting on 11 studies (Table 1). Eight studies were conducted in European countries: Finland (Saarinen, Hintikka, Vnamäki, Lehtonen, \& Lönqvist, 2000), Norway (Dyregrov, 2004; Dyregrov, Dieserud, Hjelmeland, et al., 2011), Sweden (Åsgård \& Carlsson-Bergström, 1991; Omerov et al., 2014; Runeson \& Beskow, 1991), and the UK (Cooper, 1999; Hawton et al., 2003). The other three were conducted in Canada (Henry \& Greenfield, 2009), Hong Kong (Wong et al., 2010), and Uganda (Kizza, Hjelmeland, Kinyanda, \& Knizek, 2011). Nine studies involved suicide-bereaved individuals who participated in psychological autopsy (PA) studies involving face-to-face interviews; one study used a population-based survey aiming to improve professional care for parents bereaved through suicide (Omerov et al., 2014), and one was a mixed-methods study, which included face-to-face interviews and a self-report questionnaire on psychosocial health and support (Dyregrov, 2004).

The majority $(n=8)$ of original research studies explored the experience of bereavement after a suicide death across the age range. One study explored bereavement after death by suicide, sudden infant death syndrome, or an accident in children, adolescents, and young adults (Dyregrov, 2004). Two studies did not report on the age of the deceased by suicide (Åsgård \& Carlsson-Bergström, 1991; Saarinen et al., 2000). The bereaved research participants were mostly nuclear family members, such as parents, spouses/partners, siblings, and children, as well as friends of the deceased. Other relationships, albeit in one study only (Dyregrov, Dieserud, Hjelmeland, et al., 2011), included other relatives, neighbors, and work colleagues, or were not specified. One study included adolescents (Hawton et al., 2003). Time between loss and participating in the study differed between studies and ranged between 6 weeks and 10 years, mostly between 6 months and 3 years.

Eight studies collected quantitative data on the experience of research participation, including the questionnaire-based data from the mixed-methods study (Dyregrov, 2004), and three studies collected qualitative data (Dyregrov, Dieserud, Hjelmeland, et al., 2011; Henry \& Greenfield, 2009; Kizza et al., 2011). Quantitative studies used (semi-)structured telephone interviews conducted by the original PA interviewer (Hawton et al., 2003), or another/independent researcher (Åsgård \& Carlsson-Bergström, 1991; Beskow, Runeson, \& Åsgård, 1991; Runeson \& Beskow, 1991), self-report questionnaires (Cooper, 1999; Dyregrov, 2004; Omerov et al., 2014), or a combination of questionnaire and telephone interview (Wong et al., 2010). The qualitative studies collected data through face-to-face interviews by the same interviewer immediately after the original PA interview (Dyregrov, Dieserud, Hjelmeland, et al., 2011; Henry \& Greenfield, 2009; Kizza et al., 2011). Ranging from six (Cooper, 1999; Hawton et al., 2003) to 20 (Wong et al., 2010) questions, studies have applied a varying number and combination of closed-ended and open-ended questions to inquire about the research experience. Sample questions included: "Did 


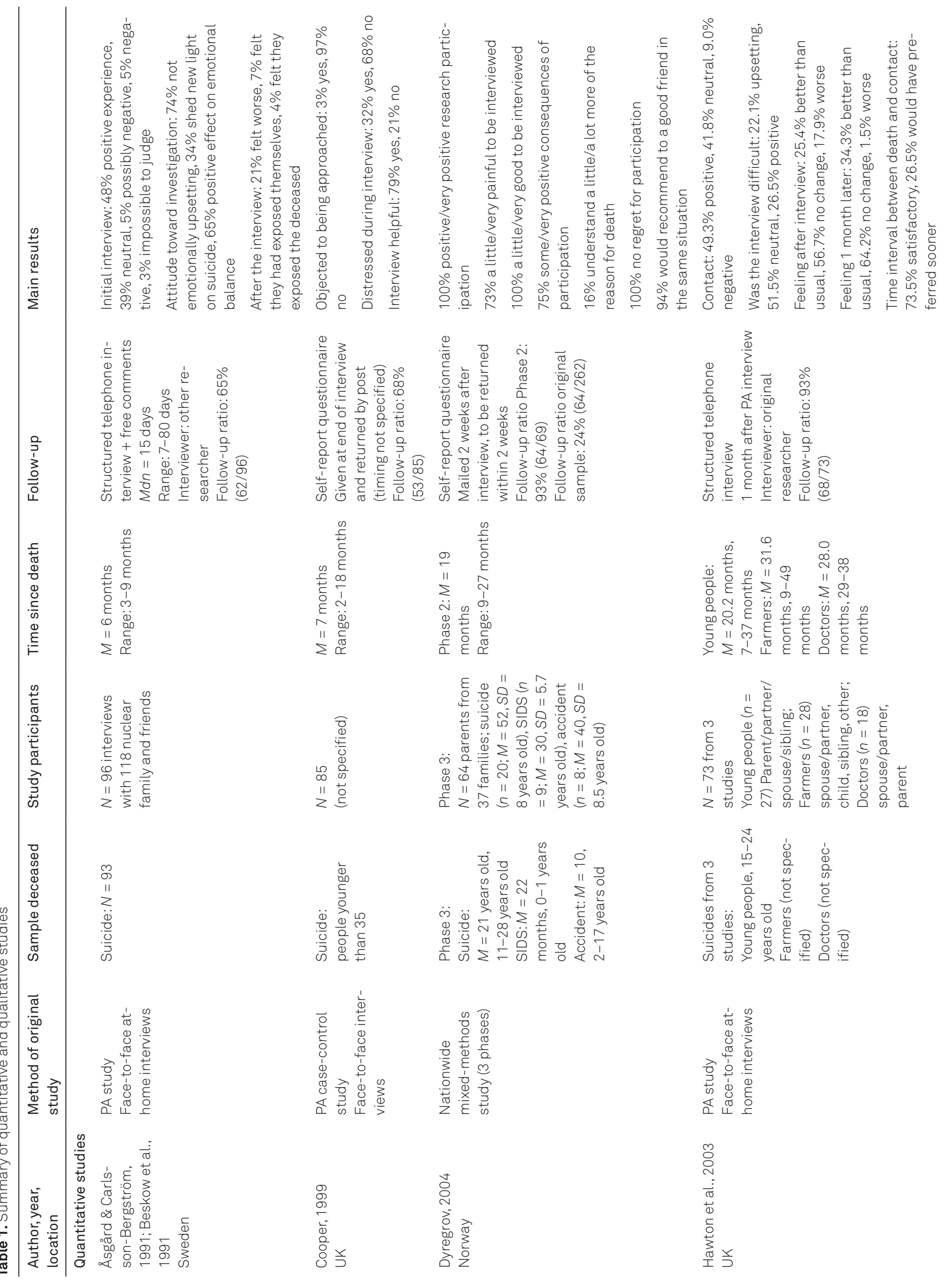




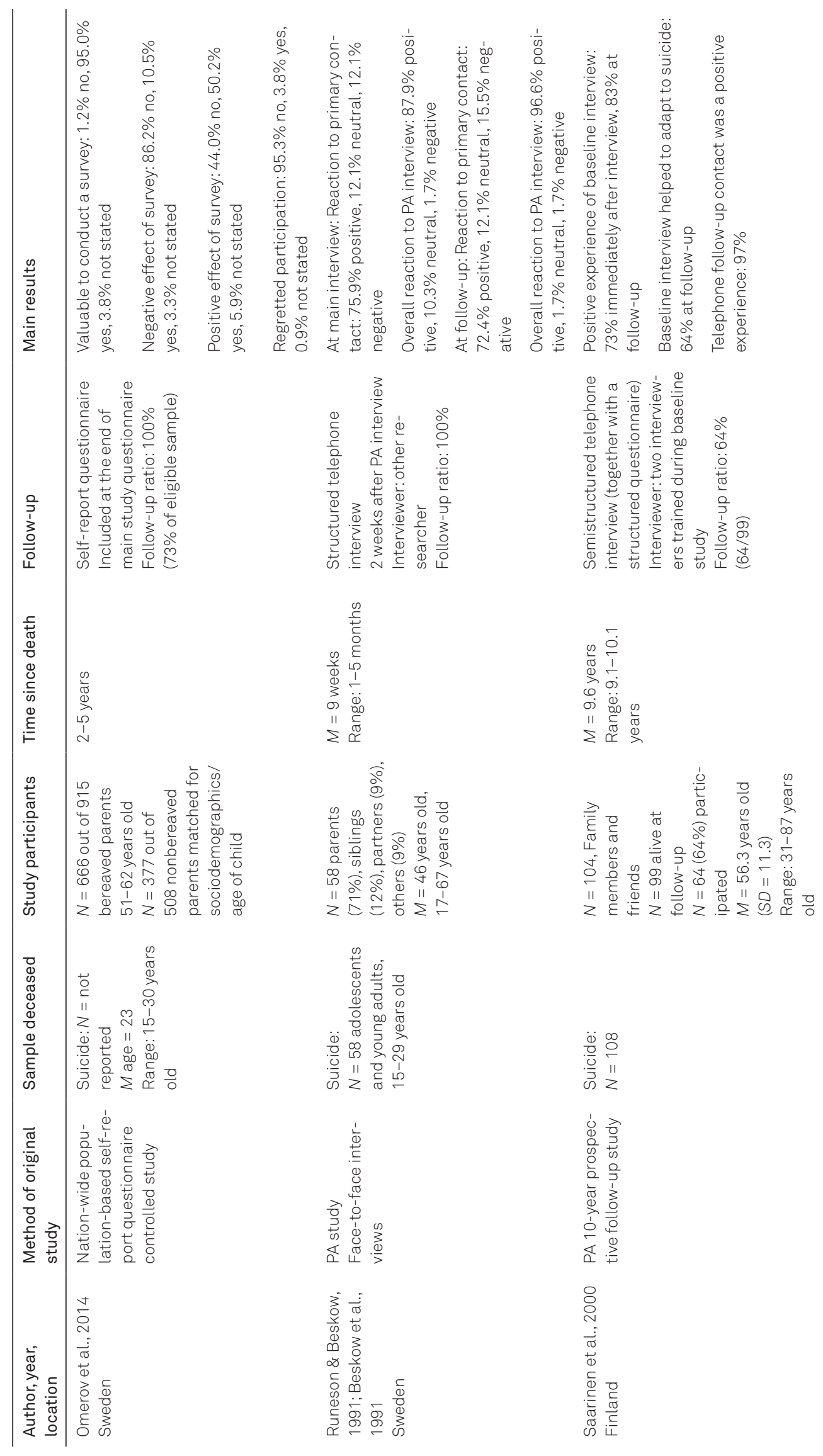




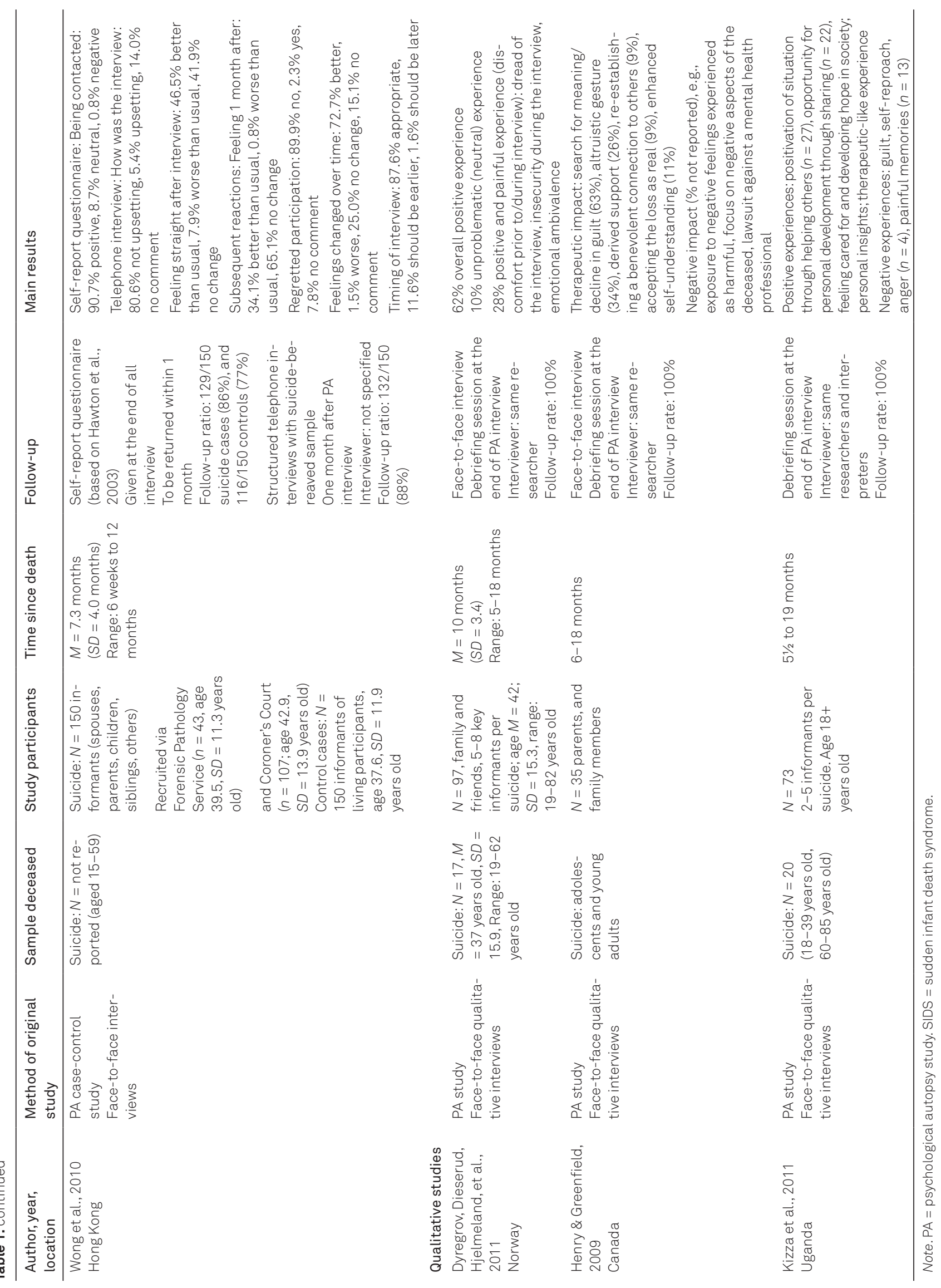


you find the interview distressing at the time?"; "Did you feel the interview had been helpful to you?" (Cooper, 1999); and: "Do you think this survey has had a negative effect on you?"; "If yes, do you think this negative effect will last?" (Omerov et al., 2014).

\section{Experience of Research Participation}

The majority of the bereaved (73-100\%) reported having an overall positive or neutral experience of research participation (Åsgård \& Carlsson-Bergström, 1991; Beskow et al., 1991; Cooper, 1999; Dyregrov, 2004; Saarinen et al., 2000), and did not regret being involved in research or would recommend participation to other bereaved individuals (89.9-100\%; Dyregrov, 2004; Omerov et al., 2014; Wong et al., 2010). A smaller proportion of participants (1.7-10\%) reported an overall negative experience (Åsgård \& Carlsson-Bergström, 1991; Beskow et al., 1991; Cooper, 1999; Dyregrov, 2004; Saarinen et al., 2000) or found the PA interview itself upsetting (5.4-22.1\%; Hawton et al., 2003; Wong et al., 2010). Of note, a positive assessment of the research experience does not imply lack of distress during the interview itself, and vice versa, having a painful interview does not preclude seeing it as a helpful experience. For instance, in the study by Dyregrov and colleagues (2004), all bereaved parents rated research participation positively, although $75 \%$ found it a little or very painful to be interviewed. Similarly, Cooper (1999) reported that although $32 \%$ of participants found the interview distressing, $79 \%$ of the group found it helpful.

Positive research experiences were related to having an opportunity to help others, to share personal experiences, to experience social support, and to re-establish a benevolent interpersonal connection (Cooper, 1999; Dyregrov, Dieserud, Hjelmeland, et al., 2011; Henry \& Greenfield, 2009; Kizza et al., 2011). Other therapeutic-like experiences reported by bereaved research participants were an opportunity to vent emotions, an enhanced capacity for self-understanding and personal insights, accepting the reality of the loss, and lessening of guilt (Dyregrov, Dieserud, Hjelmeland, et al., 2011; Henry \& Greenfield, 2009; Kizza et al., 2011; Saarinen et al., 2000). Conversely, distress or negative experiences of research participation were linked to negative feelings (guilt, self-reproach, and anger), focusing on the negative aspects of the deceased, and reactivation of painful memories (Henry \& Greenfield, 2009; Kizza et al., 2011). Saarinen et al. (2000) found that the positive evaluation of research participation could be maintained over time: $73 \%$ of the bereaved reported having a positive experience immediately after a PA interview and $83 \%$ reported a positive experience at the 10 -year follow-up.

\section{Short-Term and Long-Term Impact of Research Participation}

Hawton et al. (2003) and Wong et al. (2010) found that approximately half of participants $(56.7 \%$ and $41.9 \%$, respectively) reported no change in how they felt before and immediately after having participated in a study. The remainder felt better than usual immediately after $(25 \%$ and $47 \%$, respectively) or they felt worse $(17.9 \%$ and $7.9 \%$, respectively). The authors also explored participants' reactions 1 month after having participated in the PA study, and about two thirds (64.2\% and $65.1 \%$, respectively) of the participants reported no long-term change in how they felt before and after the interview, one third $(34.3 \%$ and $34.1 \%$, respectively) felt better than usual, and a small group ( $1.5 \%$ and $0.8 \%$, respectively) felt worse.

The negative reactions reported after having participated in a study range from feelings of sadness and regret for having exposed oneself and the deceased, depression, and struggling with painful memories, to complaints about the study methodology, such as the impersonal nature of a questionnaire or having to answer too many questions (Åsgård \& Carlsson-Bergström, 1991; Dyregrov, 2004; Omerov et al., 2014). Conversely, some participants reported being grateful for an opportunity to share the grief experiences and to talk about the deceased, or being able to help other bereaved, and to contribute to better care provision (Cooper, 1999; Omerov et al., 2014). Some bereaved participants also gained better understanding of the reason for death, and appreciated having an opportunity to work through loss-related memories and feelings (Dyregrov, 2004; Omerov et al., 2014). The majority (64\%) of the participants in the study of Saarinen et al. (2000) reported that participating in a PA study 10 years earlier had helped them adapt to the loss. In addition, some studies reported that being involved in a research study contributed to help-seeking (Beskow et al., 1991; Cooper, 1999; Hawton et al., 2003; Omerov et al., 2014; Runeson \& Beskow, 1991; Saarinen et al., 2000), and another study found no increased risk of taking sick leave or needing additional consultations with a (mental) health professional (Åsgård \& Carlsson-Bergström, 1991).

\section{Reactions to Initial Contact}

Most of the bereaved participants (88-97\%) had a positive or a neutral reaction to the initial contact and to being approached to participate in a study (Cooper, 1999; Hawton 
et al., 2003; Runeson \& Beskow, 1991; Wong et al., 2010). Hawton et al. (2003) and Wong et al. (2010) explored how participants experienced the time interval between death and initial contact. A majority of participants $(73.5 \%$ and $87.6 \%$, respectively) found it satisfactory, although between $11.6 \%$ and $26.5 \%$ would have preferred to be contacted sooner and $1.6 \%$ would have preferred to be contacted later (Wong et al., 2010). There was no association between the time interval between death and primary contact and a preference for having been contacted earlier (Hawton et al., 2003). For half (54.4\%) of the participants, timing made no difference regarding their interview/questionnaire responses, although a third (36.7\%) reported that some of their responses might have been different if they were contacted at a different time (Hawton et al., 2003). In addition, two studies explored the preferred medium of initial contact. In a study by Åsgård and Carlsson-Bergström (1991), $15 \%$ of participants would have preferred a letter before receiving a telephone call. Runeson and Beskow (1991) found that a letter was the preferred method of contact for the majority (72\%) of participants, whereas the other participants (28\%) preferred telephone contact.

\section{Factors Associated With Appraisal of Impact of Research Participation}

Dyregrov (2004) reported that female gender and a high General Health Questionnaire (GHQ; Goldberg \& Williams, 1988) score were significant predictors of negative appraisal of research participation. In a later study, Dyregrov, Dieserud, Hjelmeland, et al. (2011) found that gender, relationship to the deceased (family/not family member), suicide method, age of the informant, age of the deceased, and time between the suicide and the interview had an impact on how research participation was experienced; however, these differences did not reach the level of statistical significance. Hawton et al. (2003) observed that PA informants who had received counselling found the PA interviews more helpful than those who had not sought professional help.

\section{Processes in Place to Deal With Distressed Participants}

The studies reported in varying level of detail on their protocols on how to prevent participant distress and/or how to support distressed participants. Preventive measures included preparation and training of the interviewers, assessment of candidate interviewees in regard to potential distress, and providing information regarding the voluntary nature, the right to withdraw, and potential benefits and harms related to research participation. Some studies (Beskow et al., 1991; Dyregrov, 2004; Omerov et al., 2014; Runeson \& Beskow, 1991; Saarinen et al., 2000) used clinical scales in the assessment of interviewees, such as the GHQ (Goldberg \& Williams, 1988), and the Patient Health Questionnaire (Kroenke, Spitzer, \& Williams, 2001); none reported on suicidality. A supportive and empathic interview style, allowing the interviewee to vent negative or painful emotions, pausing the interview when necessary, and winding down at the end also aimed to prevent and minimize emotional distress. Some studies (Cooper, 1999; Dyregrov, Dieserud, Hjelmeland, et al., 2011; Henry \& Greenfield, 2009; Kizza et al., 2011) routinely offered a debriefing session or an information pack (Hawton et al., 2003) to all participants. A few participants were identified as being in psychological need after a PA interview and were referred to professional help: two participants in a study by Beskow et al. (1991) and Runeson and Beskow (1991), and two participants in a study by Kizza et al. (2011).

\section{Discussion}

This systematic review identified 12 papers reporting on 11 studies that explored the experience of research participation by people bereaved through suicide. Most participants reported a positive or a neutral experience of research participation, had no regrets, or would recommend involvement in studies to other bereaved individuals. Some studies concluded that PA interviews should be routinely offered to those bereaved through suicide (Wong et al., 2010) as they may provide support in the absence of other services (Kizza et al., 2011). The positive experiences were related to experiences of altruism (e.g., opportunities to help others), social support (e.g., to share experiences and to find interpersonal connection), and personal growth (e.g., improved insights, acceptance of the loss, and lessening of guilt) (Dyregrov, Dieserud, Hjelmeland, et al., 2011; Henry \& Greenfield, 2009; Kizza et al., 2011). For some of the bereaved, research participation was an opportunity to get acquainted with available resources (Hawton et al., 2003; Kizza et al., 2011) or facilitated help-seeking (Beskow et al., 1991; Cooper, 1999; Omerov et al., 2014; Runeson \& Beskow, 1991; Saarinen et al., 2000). Similar mechanisms have been discussed in the literature on benefits of research for vulnerable populations (Lakeman \& Fitzgerald, 2009a, 2009b; Newman \& Kaloupek, 2004), and it appears that the positive evaluation of research participation can be maintained over time (Saarinen et al., 2000).

A significant minority of the bereaved experienced research participation as negative or distressing (Hawton 
et al., 2003; Wong et al., 2010). This could be related to feelings of guilt, self-reproach and anger, focusing on the negative aspects of the deceased, and reactivation of painful memories (Henry \& Greenfield, 2009; Kizza et al., 2011). Also, sadness, regret for having exposed oneself and the deceased, depression, and complaints about the study methodology have been related to a negative research experience (Åsgård \& Carlsson-Bergström, 1991; Dyregrov, 2004; Omerov et al., 2014). Review of trauma-related research indicates that most negative feelings after research participation are short-lived and unlikely to cause long-term re-traumatization (Legerski \& Bunnell, 2010). Nevertheless, our findings confirm the need for researchers to provide accurate information about the content and the methodology of the study, and about the potential risks, benefits, and voluntary nature of participation (Moore et al., 2013).

In addition, researchers should establish a relationship based on trust and understanding, and they should conduct interviews with empathy, care (Dyregrov, 2004; Dyregrov, Dieserud, Hjelmeland, et al., 2011), and respect for the emotional boundaries of the interviewee by not forcing them to explore areas that seem troublesome (Åsgård \& Carlsson-Bergström, 1991). It is crucial to respect the interviewee's balance between what they gain (e.g., an opportunity to share painful experiences with a professional) and what they give (helping the researchers; Beskow et al., 1991; Dyregrov, 2004). Piloting the interview (Cooper, 1999) and involvement of the target group in the research design may contribute to the acceptability of the content and the method of the interview (Omerov et al., 2013, 2014). Also, focusing the interviews on selected topics rather than conducting broad-scale interviews, may limit the practical and emotional burden on interviewees (Beskow et al., 1991).

Some studies applied clinical scales to assess participants (Beskow et al., 1991; Dyregrov, 2004; Omerov et al., 2014; Runeson \& Beskow, 1991; Saarinen et al., 2000), and high GHQ scores predicted a painful research participation (Dyregrov, 2004). There is, however, no evidence that research participation in itself is traumatizing, and the reviewed studies do not allow for identification of a specific subgroup of participants for whom research participation may become too distressing. Hence, researchers should screen potential participants for vulnerabilities, not to exclude them but to take precautions (Dyregrov, 2004; Jorm et al., 2007; Omerov et al., 2014). Whereas it is crucial to avoid recruiting participants in acute distress, Dyregrov (2004) and Omerov et al. (2014) suggested that those most vulnerable and with higher psychological morbidity may decline participation to avoid additional stress, thus reinforcing the importance of accurate information being provided to potential participants.
It is noteworthy that only two studies included a control group (Omerov et al., 2014; Wong et al., 2010). Omerov et al. (2014) found that while the psychological morbidity was higher among the bereaved than the nonbereaved, motivation to participate was also higher, and no participant felt that their distress was caused by participation. Importantly, the occurrence of negative effects in the control group suggests that at least part of the effects cannot be explained through the research interview. Conversely, Wong et al. (2010) found positive effects both in the study and control group.

Most of the bereaved individuals reported either a positive or a neutral reaction to being approached to participate in a bereavement study. In general, participants found the period between death and being contacted by researchers satisfactory, although some would have preferred to be contacted sooner and others later. Bearing in mind that each study used a different time window to contact the study participants, it is conceivable that being approached to participate in a study in itself may constitute a positive experience due to the acknowledgment given to the bereaved. Hawton et al. (2003) reported that for over half of participants, timing made no difference with regard to their responses to research questions, although one third might have given other responses if they were contacted at a different time. The two studies that explored the preferred medium of initial contact found inconsistent results: Some of the participants preferred being contacted via a letter, others preferred telephone contact (Åsgård \& Carlsson-Bergström, 1991; Runeson \& Beskow, 1991). Future studies may include contact via e-mail as this may have become more common than, for example, postal letters. Similar to reactions of research participation, some of the bereaved reported a negative reaction or objected to contact. This finding highlights the importance of researchers being vigilant when contacting potential participants and of providing them with alternatives to research participation such as information and support resources.

It should be noted that although some bereaved may experience research participation as distressing or painful, they can still evaluate it positively (Cooper, 1999; Dyregrov, Dieserud, Hjelmeland, et al., 2011). While remembering the past may be upsetting, it is also a means for achieving insight - one of the most significant benefits of research participation. Jorm et al. (2007) reported a similar observation based on trauma-related and psychiatric research:

It is notable that positive reactions are largely independent of negative ones. In other words, participants who become distressed may also have a positive evaluation of the research. (...) It is therefore possible that participation could be distressing at the time, but still produce longer-term benefits. (pp. 923-924) 
Hence, distress may be only one factor among others that affect participants' judgments about the costs and benefits of the research experience (Newman \& Kaloupek, 2004). However, since one cannot regard all distressing experiences as potentially beneficial, more research in the field of suicide bereavement studies is needed to better understand this duality of painful yet beneficial experiences and how these experiences relate to long-term grief and mental health outcomes.

Studies have applied a variety of questions to investigate the research experience, and it is not known if, or how, this may have affected respondents' replies. Adaptation and usage of standardized measurements, such as the Response to Research Participation Questionnaire (Gekoski, Gray, \& Adler, 2012), may enhance the comparability of the reported data. In addition, in some studies follow-up interviews were done by the same researcher who conducted the initial PA interview (Dieserud, Hjelmeland, et al., 2011; Hawton et al., 2003; Henry \& Greenfield, 2009; Kizza et al., 2011), whereas in other studies participants were contacted by a different researcher/interviewer (Åsgård \& Carlsson-Bergström, 1991; Beskow et al., 1991; Runeson \& Beskow, 1991). These different approaches raise an issue of familiarity with the researcher, which may increase the participation rate; however, it may also introduce a response bias.

Only three studies looked at background variables mediating the experience of research participation and their results (Dyregrov, 2004; Dyregrov, Dieserud, Hjelmeland, et al., 2011; Hawton et al., 2003). Although inconclusive, these point to the importance of sociodemographic factors (gender, age of the deceased and the informant) and variables such as the type of relationship to the deceased, the suicide method, and the time between the death and the interview, as well as having received counseling. It is also possible that other participant-related factors, such as mental disorders, history of trauma and adverse life events, personality traits (such as neuroticism), and low social support, may affect the vulnerability of some research participants (Jorm et al., 2007). Also, interviewer-related variables, such as age, gender, education, and professional biases, remain unstudied (Pouliot \& De Leo, 2006). In addition, there may be differences in the psychosocial costs and impact between methods of data collection, for example, an interview or a writing task may be more psychologically demanding than paper-and-pencil instruments (Rosenbaum \& Langhinrichsen-Rohling, 2006).

\section{Limitations and Implications}

The review was limited to original studies published in peer-reviewed journals. The majority of studies (nine out of
11) looked at participation in face-to-face PA studies; only two studies focused on other methods of data collection, and only two studies included a control group. All studies were related to a larger research project; none exclusively focused on the experience of research participation. Whereas psychological autopsy studies aim to identify risk factors for suicide, most of the postvention research examines characteristics of suicide bereavement and service delivery (Andriessen, 2014). Future research may elucidate the experience of research participation in these types of studies and include control groups of nonparticipants and look at reasons for not participating in such studies with regard to sociodemographic variables, timing of contact, research methods, psychological morbidity, self-harm, and mortality. In addition, none of the reviewed studies included measures of suicide risk, although some of the individuals bereaved through suicide, including research participants, might struggle with suicidal thoughts and/or may have a history of suicidal behavior (Pitman et al., 2014). Future studies should include these variables and evaluate how research participation may impact suicide risk in this population.

No US-based research has been reported, in contrast to the majority of published suicide bereavement studies being conducted in the United States (Andriessen, 2014; Maple et al., in press). Few studies were undertaken in non-Western countries (Uganda: Kizza et al., 2011, and Hong Kong: Wong et al., 2010), highlighting the need for an international perspective on postvention research (Andriessen, Castelli Dransart, Cerel, \& Maple, 2017). The reviewed studies explored the research participation experience mostly in relation to suicide of people younger than 35 years (children, adolescents, young adults, adults). Although some studies included a wider age range of the deceased (Dyregrov, Dieserud, Hjelmeland, et al., 2011; Hawton et al., 2003; Kizza et al., 2011; Wong et al., 2010), no studies examined specifically survivors of middle-aged and older adult suicides. Also, research participants were mostly adult nuclear family members, hence it is not known if the findings extend to other bereaved relatives or non-family members.

\section{Conclusion}

This systematic review found that the majority of people bereaved through suicide evaluated research participation as a positive experience. Such participation may lead to constructive outcomes, such as gaining insight into the grief experience and providing opportunities to help others. Nonetheless, a minority objected to contact with researchers and/or reported negative experiences when participating in studies. More research is needed to better 
understand factors and mechanisms germane to participation versus nonparticipation and to the likelihood of a positive or a negative research experience. This should not be limited to PA studies, but extended to other methodologies applied in suicide bereavement research.

Future research could apply standardized measures to assess the experience of research participation and its short-term and long-term impact, including outcomes related to mental health and suicidality. Involvement of bereaved individuals in the research design also has a potential to improve participants' experience. Although the risk of lasting negative effects appears to be low, providing optimum care for all participants is essential, including offering alternatives to participation and ensuring the availability of information and support resources during and after a study. Further exploration of the experience of research participants can contribute to improved participant safety, better research design, and a stronger evidence base regarding the ethical conduct of postvention studies.

\section{Acknowledgments}

The study was supported by the Anika Foundation for Adolescent Depression and Suicide. The funding body had no role in the design of the study, the collection, analysis, and interpretation of data, the writing of the report, and the decision to submit it for publication.

The authors have no conflict of interest to report.

\section{References}

Andriessen, K. (2014). Suicide bereavement and postvention in major suicidology journals. Lessons learned for the future of postvention. Crisis, 35(5), 338-348. https://doi.org/10.1027/02275910/a000269

Andriessen, K., Castelli Dransart, D. A., Cerel, J., \& Maple, M. (2017b). Current postvention research and priorities for the future: Results of a survey. Crisis, 38(3), 202-206. https://doi. org/10.1027/0227-5910/a000459

Andriessen, K., Krysinska, K., \& Grad, O. (Eds.). (2017a). Postvention in action: The international handbook of suicide bereavement support. Göttingen, Germany: Hogrefe Publishing.

Åsgård, U., \& Carlsson-Bergström, M. (1991). Interviews with survivors of suicides: Procedures and follow-up of interview subjects. Crisis, 12(1), 21-33.

Beskow, J., Runeson, B., \& Åsgård, U. (1991). Ethical aspects of psychological autopsy. Acta Psychiatrica Scandinavica, 84(5), 482-487. https://doi.org/10.1111/j.1600-0447.1991.tb03181.x

Buckle, J. L., Dwyer, S. C., \& Jackson, M. (2010). Qualitative bereavement research: Incongruity between the perspectives of participants and research ethics boards. International Journal of Social Research Methodology, 13(2), 111-125. https://doi. org/10.1080/13645570902767918

Castelli Dransart, D. A. (2017). Reclaiming and reshaping life: Patterns of reconstruction after the suicide of a loved one. Qualitative Health Research, 27(7), 994-1005. https://doi.org/ $10.1177 / 1049732316637590$
Commonwealth of Australia. (2015). National statement on ethical conduct in human research 2007 (updated May 2015). Canberra, Australia: The National Health and Medical Research Council, the Australian Research Council and the Australian Vice-Chancellors' Committee.

Cook, A. S., \& Bosley, G. (1995). The experience of participating in bereavement research:Stressful or therapeutic?. Death Studies, 19(2), 157-170. https://doi.org/10.1080/07481189508252722

Cooper, J. (1999). Ethical issues and their practical application in a psychological autopsy study of suicide. Journal of Clinical Nursing, 8(4), 467-475. https://doi.org/10.1046/j.13652702.1999.00276.x

Dazzi, T., Gribble, R., Wessely, S., \& Fear, N. T. (2014). Does asking about suicide and related behaviours induce suicidal ideation? What is the evidence? Psychological Medicine, 44(16), 33613363. https://doi.org/10.1017/S0033291714001299

DeCou, C. R., \& Schumann, M. E. (2017). On the iatrogenic risk of assessing suicidality: A met-analysis. Suicide and Life-Threatening Behavior. Advance online publication. https://doi.org/10.1111/ sltb.12368

Dyregrov, K. (2004). Bereaved parents' experience of research participation. Social Science and Medicine, 58(2), 391-400. https:// doi.org/10.1016/S0277-9536(03)00205-3

Dyregrov, K. M., Dieserud, G., Hjelmeland, H. M., Straiton, M., Rasmussen, M. L., Knizek, B. L., \& Leenaars, A. A. (2011). Meaning-making through psychological autopsy interviews: The value of participating in qualitative research for those bereaved by suicide. Death Studies, 35(8), 685-710. https://doi.org/10.1080/ 07481187.2011 .553310

Dyregrov, K., Dieserud, G., Straiton, M., Rasmussen, M. L., Hjelmeland, H., Knizek, B. L., \& Leenaars, A. A. (2011). Motivation for research participation among people bereaved by suicide. Omega: Journal of Death and Dying, 62(2), 149-168. https://doi. org/10.2190/OM.62.2.d

Feigelman, W., Jordan, J. R., McIntosh, J. L., \& Feigelman, B. (2012). Devastating losses: How parents cope with the death of a child to suicide or drugs. New York, NY: Springer.

Gekoski, A., Gray, J. M., \& Adler, J. R. (2012). Interviewing women bereaved by homicide: Assessing the impact of trauma-focused research. Psychology, Crime \& Law, 18(2), 177-189. https://doi. org/10.1080/10683161003718692

Genest, C., Moore, M., \& Nowicke, C. (2017). Posttraumatic growth after suicide. In K. Andriessen, K. Krysinska, \& O. Grad (Eds.), Postvention in action: The international handbook of suicide bereavement support (pp. 50-59). Göttingen, Germany: Hogrefe Publishing.

Goldberg, D., \& Williams, P. (1988). User's guide to the general health questionnaire. Windsor, UK: NFER-Nelson.

Grad, O., \& Andriessen, K. (2016). Surviving the legacy of suicide. In R. O'Connor \& J. Pirkis (Eds.), International handbook of suicide prevention (2nd ed., pp. 663-680). Chichester, UK: Wiley-Blackwell.

Gysels, M. H., Evans, C., \& Higginson, I. J. (2012). Patient, caregiver, health professional and researcher views and experiences of participating in research at the end of life: A critical interpretive synthesis of the literature. BMC Medical Research Methodology, 12(1), 123. https://doi.org/10.1186/1471-2288-12-123

Hawton, K., Houston, K., Malmbergand, A., \& Simkin, S. (2003). Psychological autopsy interviews in suicide research: The reactions of informants. Archives of Suicide Research, 7(1), 73-82. https:// doi.org/10.1080/13811110301566

Henry, M., \& Greenfield, B. J. (2009). Therapeutic effects of psychological autopsies: The impact of investigating suicides on interviewees. Crisis, 30(1), 20-24. https://doi.org/10.1027/02275910.30 .1 .20 
Hom, M. A., Podlogar, M. C., Stanley, I. H., \& Joiner, T. E., Jr. (2016). Ethical issues and practical challenges in suicide research. Crisis, 38(2), 107-114. https://doi.org/10.1027/0227-5910/a000415

Hynson,J.L.,Aroni, R., Bauld,C., \& Sawyer,S.M.(2006). Research with bereaved parents: A question of how not why. Palliative Medicine, 20(8), 805-811. https://doi.org/10.1177/0269216306072349

Jorm, A. F., Kelly, C. M., \& Morgan, A. J. (2007). Participant distress in psychiatric research: A systematic review. Psychological Medicine, 37(7), 917-926. https://doi.org/10.1017/S0033 291706009779

Kizza, D., Hjelmeland, H., Kinyanda, E., \& Knizek, B. L. (2011). Qualitative psychological autopsy interviews on suicide in post-conflict Northern Uganda: The participants' perceptions. Omega: Journal of Death and Dying, 63(3), 235-254. https://doi. org/10.2190/OM.63.3.c

Kroenke, K., Spitzer, R. L., \& Williams, J. B. (2001). The PHQ-9: Validity of a brief depression severity measure. Journal of General Internal Medicine, 16(9), 606-613. https://doi.org/10.1046/ j.1525-1497.2001.016009606.x

Lakeman, R., \& Fitzgerald, M. (2009a). The ethics of suicide research: The views of ethics committee members. Crisis, 30(1), 13-19. https://doi.org/10.1027/0227-5910.30.1.13

Lakeman, R., \& Fitzgerald, M. (2009b). Ethical suicide research:A survey of researchers. International Journal of Mental Health Nursing, 18(1), 10-17. https://doi.org/10.1111/j.1447-0349.2008.00569.x

Legerski, J. P., \& Bunnell, S. L. (2010). The risks, benefits, and ethics of trauma-focused research participation. Ethics \& Behavior, 20(6), 429-442. https://doi.org/10.1080/10508422.2010.521443

Litman, R., Curphey,T., Shneidman, E., Farberow, N. L., \& Tabachnick, N. (1963). Investigations of equivocal suicides. JAMA, 184(12), 924-929. https://doi.org/10.1001/jama.1963.03700250060008

Maple, M., Pearce, T., Sanford, R., Cerel, J., Castelli Dransart, D. A., \& Andriessen, K. (in press). A systematic mapping of suicide bereavement and postvention research and a proposed strategic research agenda. Crisis. https://doi.org/10.1027/0227-5910/ a000498

McClinton Appollis, T., Lund, C., de Vries, P.J., \& Mathews, C. (2015). Adolescents' and adults' experiences of being surveyed about violence and abuse: A systematic review of harms, benefits, and regrets. American Journal of Public Health, 105(2), e31-e45. https://doi.org/10.2105/AJPH.2014.302293

Moher, D., Liberati, A., Tetzlaff, J., \& Altman, D. G. (2009). Preferred reporting items for systematic reviews and meta-analyses: The PRISMA statement. Annals of Internal Medicine, 151(4), 264-269. https://doi.org/10.7326/0003-4819-151-4-200908180-00135

Moore, M., Maple, M., Mitchell, A. M., \& Cerel, J. (2013). Challenges and opportunities for suicide bereavement research. Crisis, 34(5), 297-304. https://doi.org/10.1027/0227-5910/a000191

Neimeyer, R., \& Sands, D. (2017). Suicide loss and the quest for meaning. In K. Andriessen, K. Krysinska, \& O. Grad (Eds.), Postvention in action: The international handbook of suicide bereavement support (pp. 71-84). Göttingen, Germany: Hogrefe Publishing.

Newman, E., \& Kaloupek, D. G. (2004). The risks and benefits of participating in trauma-focused research studies. Journal of Traumatic Stress, 17(5), 383-394. https://doi.org/10.1023/B:JOTS.0000048951.02568.3a

Omerov, P., Steineck, G., Dyregrov, K., Runeson, B., \& Nyberg, U. (2014). The ethics of doing nothing. Suicide-bereavement and research: Ethical and methodological considerations. Psychological Medicine, 44(16), 3409-3420. https://doi.org/10.1017/ S0033291713001670

Omerov, P., Steineck, G., Runeson, B., Christensson, A., Kreicbergs, U., Pettersén, R., ... Nyberg, U. (2013). Preparatory studies to a population-based survey of suicide-bereaved parents in Swe- den. Crisis, 34(3), 200-210. https://doi.org/10.1027/0227-5910/ a000175

Pitman, A., Osborn, D., King, M., \& Erlangsen, A. (2014). Effects of suicide bereavement on mental health and suicide risk. Lancet Psychiatry, 1(1), 86-94. https://doi.org/10.1016/S22150366(14)70224-X

Pouliot, L., \& De Leo, D. (2006). Critical issues in psychological autopsy studies. Suicide and Life-Threatening Behavior, 36(5), 491-510. https://doi.org/10.1521/suli.2006.36.5.491

Rosenbaum, A., \& Langhinrichsen-Rohling, J. (2006). Meta-research on violence and victims: The impact of data collection methods on findings and participants. Violence and Victims, 21(4), 404-409.

Runeson, B., \& Beskow, J. (1991). Reactions of survivors of suicide victims to interviews. Acta Psychiatrica Scandinavica, 83(3), 169-173. https://doi.org/10.1111/j.1600-0447.1991.tb05518.x

Saarinen, P. I., Hintikka, J., Vnamäki, H., Lehtonen, J., \& Lönqvist, J. (2000). Is it possible to adapt to the suicide of a close individual? Results of a 10-year prospective follow-up study. International Journal of Social Psychiatry, 46(3), 182-190. https://doi. org/10.1177/002076400004600304

Scarth, B., \& Schafer, C. (2016). Resilient vulnerabilities: Bereaved persons discuss their experience of participating in thanatology research. In M. Tolich (Ed.), Qualitative ethics in practice (pp. 85-95). New York, NY: Routledge.

Supiano, K. P. (2012). Sense-making in suicide survivorship: A qualitative study of the effect of grief support group participation. Journal of Loss and Trauma, 17(6), 489-507. https://doi.org/ 10.1080/15325024.2012.665298

Wilson, A. (2010). Consumer participation: Ensuring suicide postvention research counts for end users. International Journal of Nursing Practice, 16(1), 7-13. https://doi.org/10.1111/j.1440172X.2009.01811.X

Wong, P. W., Chan, W. S., Beh, P. S., Yau, F. W., Yip, P. S., \& Hawton, K. (2010). Research participation experiences of informants of suicide and control cases. Crisis, 31(5), 238-246. https://doi. org/10.1027/0227-5910/a000025

World Health Organization. (2014). Preventing suicide: A global imperative. Geneva, Switzerland: Author.

World Medical Association. (2013). WMA Declaration of Helsinki: Ethical principles for medical research involving human subjects 2013. Retrieved from https://www.wma.net/policies-post/ wma-declaration-of-helsinki-ethical-principles-for-medical-research-involving-human-subjects/

Received July 14, 2017

Revision received November 23, 2017

Accepted November 26, 2017

Published online April 5, 2018

Karl Andriessen, MSuicidology, is a PhD candidate at the School of Psychiatry, University of New South Wales, Australia, supported by the Anika Foundation for Adolescent Depression and Suicide. He is affiliated with the Faculty of Psychology and Educational Sciences, KU Leuven, Belgium. His research focuses on suicide prevention, bereavement, and postvention.

Karolina Krysinska, PhD, MA, is a research fellow at the Centre for Primary Health Care and Equity, University of New South Wales, Australia. She is an affiliated researcher with the Faculty of Psychology and Educational Sciences, KU Leuven, Belgium. Her research is focused on suicide prevention, postvention, mental health, and trauma. 
Brian Draper is Conjoint Professor, School of Psychiatry, University of New South Wales, Sydney, Australia. Dr. Draper is Senior Staff Specialist Psychiatrist and Clinical Lead, Academic Department for Old Age Psychiatry, Prince of Wales Hospital, Randwick, Australia.

Michael Dudley is Senior Staff Specialist in Psychiatry at Prince of Wales and Sydney Children's Hospitals, and Conjoint Senior Lecturer in Psychiatry at the University of New South Wales, Australia. His research and practice commitments mainly concern suicide prevention and vulnerable populations.

Philip B. Mitchell, AM, FAHMS, FASSA, MB BS, MD, FRANZCP, FRCPsych, is Scientia Professor and Head of the School of Psychiatry at the University of New South Wales, Sydney, Australia. His research interests focus on depression and bipolar disorder.

\section{Karl Andriessen}

UNSW School of Psychiatry Black Dog Institute Building Hospital Road

Randwick NSW 2031

Australia

k.andriessen@unsw.edu.au karl.andriessen@gmail.com 